\title{
THE DERIVED-DISCRETE ALGEBRAS AND STANDARD EQUIVALENCES
}

\author{
XIAO-WU CHEN, CHAO ZHANG*
}

\begin{abstract}
We prove that any derived equivalence between derived-discrete algebras of finite global dimension is standard, that is, isomorphic to the derived tensor functor by a two-sided tilting complex.
\end{abstract}

\section{INTRODUCTION}

Let $k$ be an algebraically closed field. For a finite dimensional $k$-algebra $A$, we denote by $A$-mod the category of finite dimensional $A$-modules and by $\mathbf{D}^{b}(A$-mod) the bounded derived category. By a derived equivalence between two algebras $A$ and $B$, we mean a $k$-linear triangle equivalence between $\mathbf{D}^{b}(A$-mod $)$ and $\mathbf{D}^{b}(B$-mod).

It is an open question in [14 whether any derived equivalence between two algebras is standard, that is, isomorphic to the derived tensor functor by a two-sided tilting complex. The question is answered affirmatively for hereditary algebras [13, (anti-)Fano algebras [12, triangular algebras [6] and the algebra of dual numbers [8].

The main result of this paper answers the open question affirmatively for deriveddiscrete algebras of finite global dimension; see Theorem 3.6. Recall from [8] that the open question is affirmative for an algebra $A$, provided that the category $A$-proj of finite dimensional projective $A$-modules is $\mathbf{K}$-standard in the sense of 8 . Then the main result boils down to the following result: the category $A(r, N)$-proj is $\mathbf{K}$-standard, where $A(r, N)$ is a certain Nakayama algebra given by a cyclic quiver with $N$ vertices and $r$ consecutive quadratic zero relations; see Theorem 3.5

Derived-discrete algebras are introduced in [15. They form an important class of algebras for testing conjectures and carrying out concrete calculation, since their bounded derived categories are very much accessible. We mention that we rely on the classification [4] of derived-discrete algebras up to derived equivalence.

We draw an immediate consequence of Theorem 3.6 the group of standard derived autoequivalences for a derived-discrete algebra of finite global dimension, as calculated in [10, 5], coincides with the whole group of derived autoequivalences.

The paper is structured as follows. In Section 2, we recall basic facts on triangle functors and determine the centers of a certain triangulated category. In Section 3, we formulate Theorem 3.5, from which we deduce Theorem 3.6. In Section 4, we prove Theorem 3.5 for the case $r=1$. In Section 5, we sketch the proof for the case $r>1$, since it is very close to the one in Section 4 . We mention that the argument here is similar to, but more complicated than, the one in [ 8 , Section 7].

Date: March 21, 2022.

2010 Mathematics Subject Classification. 18E30, 16G10, 18G35, 16E05.

Key words and phrases. derived-discrete algebra, standard equivalence, K-standard category.

* The corresponding author.

E-mail: xwchen@mail.ustc.edu.cn, czhang2@gzu.edu.cn. 


\section{TRIANGLE FUnCTORS AND CENTERS}

In this section, we first recall from [8] some facts on triangle functors and the centers of a triangulated category. We determine the centers of a certain triangulated category. For triangulated categories, we refer to 9,16 .

Let $\mathcal{T}$ and $\mathcal{T}^{\prime}$ be triangulated categories, whose suspension functors are denoted by $\Sigma$ and $\Sigma^{\prime}$, respectively. A triangle $(F, \omega): \mathcal{T} \rightarrow \mathcal{T}^{\prime}$ consists of an additive functor $F$ with a natural isomorphism $\omega: F \Sigma \rightarrow \Sigma^{\prime} F$, which sends an exact triangle to an exact triangle. More precisely, an exact triangle $X \stackrel{f}{\rightarrow} Y \stackrel{g}{\rightarrow} Z \stackrel{h}{\rightarrow} \Sigma(X)$ in $\mathcal{T}$ is sent to an exact triangle $F(X) \stackrel{F(f)}{\rightarrow} F(Y) \stackrel{F(g)}{\rightarrow} F(Z) \stackrel{\omega_{X} \circ F(h)}{\rightarrow} \Sigma^{\prime}(F X)$ in $\mathcal{T}^{\prime}$. We call $\omega$ the connecting isomorphism for $F$. We sometimes suppress $\omega$ and say that $F$ is a triangle functor. For example, the identity functor $\left(\operatorname{Id}_{\mathcal{T}}, \operatorname{Id}_{\Sigma}\right): \mathcal{T} \rightarrow \mathcal{T}$ is a triangle functor, which is often abbreviated as $\operatorname{Id}_{\mathcal{T}}$. A natural transformation $\eta:(F, \omega) \rightarrow\left(F_{1}, \omega_{1}\right)$ between triangle functors is a natural transformation respecting the connecting isomorphisms, that is, $\omega_{1} \circ \eta \Sigma=\Sigma^{\prime} \eta \circ \omega$.

The following well-known fact is given in [8, Lemma 2.3].

Lemma 2.1. Let $(F, \omega): \mathcal{T} \rightarrow \mathcal{T}^{\prime}$ be a triangle functor. Suppose that we are given an isomorphism $\delta_{X}: F(X) \rightarrow F_{1}(X)$ in $\mathcal{T}^{\prime}$ for each object $X \in \mathcal{T}$. Then there is a unique triangle functor $\left(F_{1}, \omega_{1}\right)$ such that $\delta:(F, \omega) \rightarrow\left(F_{1}, \omega_{1}\right)$ is an isomorphism between triangle functors.

We will call the given isomorphisms $\delta_{X}$ 's the adjusting isomorphisms, and say that the new triangle functor $\left(F_{1}, \omega_{1}\right)$ is adjusted from $(F, \omega)$.

Proof. The functor $F_{1}$ necessarily acts on a morphism $f: X \rightarrow Y$ by $\delta_{Y} \circ F(f) \circ$ $\left(\delta_{X}\right)^{-1}$, and the connecting isomorphism $\omega_{1}$ is given by $\left(\Sigma^{\prime} \delta\right) \circ \omega \circ(\delta \Sigma)^{-1}$.

In what follows, we fix a field $k$. The triangulated category $\mathcal{T}$ is required to be $k$-linear, which is Hom-finite and Krull-Schmidt. All the functors will be assumed to be $k$-linear.

For a set $\mathcal{M}$ of morphisms in $\mathcal{T}$, we denote by $\operatorname{obj}(\mathcal{M})$ the full subcategory formed by those objects, which are either the domain or the codomain of a morphism in $\mathcal{M}$. We say that $\mathcal{M} k$-linearly spans $\mathcal{T}$, provided that each morphism in $\operatorname{obj}(\mathcal{M})$ is a $k$-linear combination of the identity morphisms and composition of morphisms from $\mathcal{M}$, and that each object in $\mathcal{T}$ is isomorphic to a finite direct sum of objects in $\operatorname{obj}(\mathcal{M})$.

The following fact follows from [8, Lemmas 2.5 and 2.3].

Lemma 2.2. Let $\mathcal{M}$ be a spanning set of morphisms in $\mathcal{T}$. Assume that $(F, \omega): \mathcal{T} \rightarrow$ $\mathcal{T}$ is a $k$-linear triangle endofunctor such that $F(f)=f$ for each $f \in \mathcal{M}$. Then there is a unique natural isomorphism $\omega^{\prime}: \Sigma \rightarrow \Sigma$ and a unique natural isomorphism $\theta:(F, \omega) \rightarrow\left(\operatorname{Id}_{\mathcal{T}}, \omega^{\prime}\right)$ between triangle functors satisfying $\theta_{S}=\operatorname{Id}_{S}$ for each object $S$ from obj $(\mathcal{M})$.

We denote by $Z(\mathcal{T})$ the center of $\mathcal{T}$, which consists of all natural transformations $\lambda: \operatorname{Id}_{\mathcal{T}} \rightarrow \operatorname{Id}_{\mathcal{T}}$. Then $Z(\mathcal{T})$ is a commutative $k$-algebra, whose multiplication is induced by the composition of natural transformations. We denote by $Z_{\Delta}(\mathcal{T})$ the triangle center, which is a subalgebra of $Z(\mathcal{T})$ consisting of natural transformations $\lambda: \operatorname{Id}_{\mathcal{T}} \rightarrow \operatorname{Id}_{\mathcal{T}}$ between triangle functors. Then $\lambda \in Z(\mathcal{T})$ belongs to $Z_{\Delta}(\mathcal{T})$ if and only if $\Sigma\left(\lambda_{X}\right)=\lambda_{\Sigma(X)}$ for any object $X \in \mathcal{T}$.

The center is related to almost-vanishing endomorphisms; see [11. Let $X$ be an indecomposable object in $\mathcal{T}$. An nonzero non-invertible endomorphism $\Delta: X \rightarrow X$ is almost-vanishing provided that $\Delta \circ f=0=g \circ \Delta$ for any non-retraction morphism $f: Y \rightarrow X$ and non-section $g: X \rightarrow Z$. We observe that $\Delta$ is almost-vanishing if 
and only if it fits into an almost split triangle $\Sigma^{-1}(X) \rightarrow E \rightarrow X \stackrel{\Delta}{\rightarrow} X$; see $\underline{9}$, I.4.1]. Here, we identity $X$ with $\Sigma\left(\Sigma^{-1} X\right)$. Moreover, we have $\Delta^{2}=0$.

We denote by ind $\mathcal{T}$ a complete set of representatives of isoclasses of indecomposable objects in $\mathcal{T}$. We observe that a natural transformation $\eta: F \rightarrow F_{1}$ between endofunctors on $\mathcal{T}$ is uniquely determined by its restriction on ind $\mathcal{T}$.

The following construction of central elements is due to [11, Lemma 2.2]; see also [8, Lemma 2.7].

Lemma 2.3. Assume that $X \in \operatorname{ind} \mathcal{T}$ has an almost-vanishing endomorphism $\Delta$. Then there is a unique element $\delta(X) \in Z(\mathcal{T})$ satisfying $\delta(X)_{X}=\Delta$ and $\delta(X)_{Y}=0$ for any $Y \in \operatorname{ind} \mathcal{T}$, different from $X$.

The following fact, due to [8, Proposition 2.6], will be used often.

Lemma 2.4. Assume that $X \stackrel{f}{\rightarrow} Y \stackrel{g}{\rightarrow} Z \stackrel{h}{\rightarrow} \Sigma(X)$ is an exact triangle in $\mathcal{T}$ with $g \neq 0$ and $h \neq 0$ such that $\operatorname{End}_{\mathcal{T}}(Z)$ either equals $k \operatorname{Id}_{Z}$ or $k \operatorname{Id}_{Z} \oplus k \Delta$, where the endomorphism $\Delta$ on $Z$ is almost-vanishing. Then for a nonzero scalar $\lambda \in k$, the triangle $X \stackrel{f}{\rightarrow} Y \stackrel{g}{\rightarrow} Z \stackrel{\lambda h}{\longrightarrow} \Sigma(X)$ is exact if and only if $\lambda=1$.

We will consider the following condition on $\mathcal{T}$.

(A1) There is a disjoint union ind $\mathcal{T}=S \cup S^{\prime}$ such that $\operatorname{End} \mathcal{T}(X)=k \operatorname{Id}_{X} \oplus k \Delta_{X}$ for each $X \in S$ with $\Delta_{X}$ a fixed almost-vanishing endomorphism on $X$, and that $\operatorname{End}_{\mathcal{T}}(Y)=k \operatorname{Id}_{Y}$ for each $Y \in S^{\prime}$.

The triangulated category $\mathcal{T}$ is a block, provided that $\mathcal{T}$ does not admit a decomposition into the product of two nonzero triangulated subcategories. It is non-degenerate if there is a nonzero non-invertible morphism $X \rightarrow Y$ for some $X, Y \in \operatorname{ind} \mathcal{T}$; for details, see [7, Section 4].

Proposition 2.5. Let $\mathcal{T}$ be a non-degenerate block satisfying (A1). Then the following statements hold.

(1) $Z(\mathcal{T})=k 1 \oplus\left(\prod_{X \in S} \delta(X)\right)$, where $\prod_{X \in S} \delta(X)$ is an ideal of square zero.

(2) Let $\lambda \in Z(\mathcal{T})$ be invertible. Then $\left(\operatorname{Id}_{\mathcal{T}}, \Sigma(\lambda)\right)$ is a triangle functor if and only if $1-\lambda$ lies in $\prod_{X \in S} \delta(X)$.

Here, the element $\delta(X) \in Z(\mathcal{T})$ is obtained by applying Lemma 2.3 to the fixed almost-vanishing endomorphism $\Delta_{X}$ for each $X \in S$. We observe that if $\left(\operatorname{Id}_{\mathcal{T}}, \omega\right)$ is a triangle functor, then there is a unique invertible element $\lambda \in Z(\mathcal{T})$ with $\omega=\Sigma(\lambda)$. Hence, statement (2) characterizes all such connecting isomorphisms $\omega$.

Proof. Let $\lambda \in Z(\mathcal{T})$. We assume that $\lambda_{X}=c(X) \operatorname{Id}_{X}+b(X) \Delta_{X}$ for $X \in S$ and $\lambda_{Y}=c(Y) \operatorname{Id}_{Y}$ for $Y \in S^{\prime}$, where $c(X), b(X)$ and $c(Y)$ are scalars. For (1), it suffices to claim that the function $c$ is constant on $\operatorname{ind} \mathcal{T}$.

We first observe that if $\operatorname{Hom}_{\mathcal{T}}(U, V) \neq 0$ for $U, V \in \operatorname{ind} \mathcal{T}$, we have $c(U)=c(V)$. We just apply the naturality of $\lambda$ to the nonzero morphism $U \rightarrow V$. Here, if $U$ or $V$ lies in $S$, we use the fact that $\Delta_{U}$ or $\Delta_{V}$ is almost-vanishing. Now the claim follows by combining the observation and the following fact: for any indecomposables $U$ and $V$, there is a sequence $U=X_{0}, X_{1}, \cdots, X_{n}=V$ of indecomposables, such that $\operatorname{Hom}_{\mathcal{T}}\left(X_{i}, X_{i+1}\right) \neq 0$ or $\operatorname{Hom}_{\mathcal{T}}\left(X_{i+1}, X_{i}\right) \neq 0$; see [7, Proposition 4.2 and Remark 4.7].

For (2), it suffices to claim that $\left(\operatorname{Id}_{\mathcal{T}}, \Sigma(\lambda)\right)$ is a triangle functor if and only if the constant function $c$ has value 1 .

For the "only if" part, we take a nonzero non-invertible morphism $h: U \rightarrow \Sigma(V)$ for some indecomposables $U$ and $V$, since $\mathcal{T}$ is non-degenerate. Form an exact 
triangle $V \stackrel{f}{\rightarrow} E \stackrel{g}{\rightarrow} U \stackrel{h}{\rightarrow} \Sigma(V)$. Applying the triangle functor $\left(\operatorname{Id}_{\mathcal{T}}, \Sigma(\lambda)\right)$ to this triangle, we obtain another exact triangle

$$
V \stackrel{f}{\longrightarrow} E \stackrel{g}{\longrightarrow} U \stackrel{c(V) h}{\longrightarrow} \Sigma(V) .
$$

Here, we use the fact $\Sigma\left(\Delta_{V}\right) \circ h=0$, in case $V$ lies in $S$. By Lemma 2.4, we infer that $c(V)=1$.

For the "if" part, we take an arbitrary exact triangle

$$
\xi: X \stackrel{a}{\longrightarrow} Y \stackrel{b}{\longrightarrow} Z \stackrel{u}{\longrightarrow} \Sigma(X)
$$

in $\mathcal{T}$. We observe that $u$ is isomorphic to $u^{\prime} \oplus \operatorname{Id}_{\Sigma(K)}$ for a radical morphism $u^{\prime}: Z^{\prime} \rightarrow \Sigma\left(X^{\prime}\right)$ and some object $K$. Then $\xi$ is isomorphic to the direct sum of $\xi^{\prime}: X^{\prime} \stackrel{a^{\prime}}{\rightarrow} Y \stackrel{b^{\prime}}{\rightarrow} Z^{\prime} \stackrel{u^{\prime}}{\rightarrow} \Sigma\left(X^{\prime}\right)$ and the trivial triangle $\xi^{\prime \prime}: K \rightarrow 0 \rightarrow \Sigma(K) \stackrel{\operatorname{Id}_{\Sigma(K)}}{\longrightarrow}$ $\Sigma(K)$. We apply $\left(\operatorname{Id}_{\mathcal{T}}, \Sigma(\lambda)\right)$ to $\xi^{\prime}$. Then the resulted triangle coincides with $\xi^{\prime}$, since we have $\Sigma\left(\lambda_{X^{\prime}}\right) \circ u^{\prime}=u^{\prime}$, using the fact that the morphism $u^{\prime}$ is radical. The trivial triangle $\xi^{\prime \prime}$ stays trivial by applying $\left(\operatorname{Id}_{\mathcal{T}}, \Sigma(\lambda)\right)$. It follows that the resulted triangle by applying $\left(\operatorname{Id}_{\mathcal{T}}, \Sigma(\lambda)\right)$ to $\xi$ is exact, that is, $\left(\operatorname{Id}_{\mathcal{T}}, \Sigma(\lambda)\right)$ is a triangle functor.

We need a further condition to determine the triangle center.

(A2) We assume (A1). Moreover, we assume that the action of $\Sigma$ on $S$ is free, that is, for each $X \in S$ and $n \geq 1, \Sigma^{n}(X) \simeq X$ implies $n=1$.

We denote by $S / \Sigma$ the set of $\Sigma$-orbits in $S$. Since each orbit is infinite, we can always make the following choice: for each $X \in S$, we choose an isomorphism

$$
t_{X}: \Sigma(X) \longrightarrow X^{\prime}
$$

for a uniquely determined $X^{\prime} \in S$; moreover, we assume that

$$
\Delta_{X^{\prime}}=t_{X} \circ \Sigma\left(\Delta_{X}\right) \circ t_{X}^{-1} \text {. }
$$

For the latter assumption, we adjust the almost-vanishing endomorphisms in (A1).

For $X \in S$, we denote by $[X]$ its $\Sigma$-orbit. We denote by $\delta([X]) \in Z(\mathcal{T})$, which is uniquely determined by $\delta([X])_{Y}=0$ for $Y \in \operatorname{ind} \mathcal{T} \backslash[X]$ and $\delta([X])_{Z}=\Delta_{Z}$ for $Z \in[X]$. Indeed, by the identification in Proposition $2.5(1), \delta([X])$ corresponds to the formal sum $\sum_{Z \in[X]} \delta(Z)$.

Proposition 2.6. Let $\mathcal{T}$ be a non-degenerate block satisfying (A2). We keep the choices (2.1) and (2.2). Then the following statements hold.

(1) $Z_{\Delta}(\mathcal{T})=k 1 \oplus\left(\prod_{[X] \in S / \Sigma} \delta([X])\right)$, where $\prod_{[X] \in S / \Sigma} \delta([X])$ is an ideal of square zero.

(2) Each triangle functor $\left(\operatorname{Id}_{\mathcal{T}}, \omega\right)$ is isomorphic to $\left(\operatorname{Id}_{\mathcal{T}}, \operatorname{Id}_{\Sigma}\right)$.

Proof. Take $\lambda \in Z_{\Delta}(\mathcal{T})$. By Proposition 2.5(1), we may assume

$$
\lambda=c+\sum_{X \in S} b(X) \delta(X)
$$

for $c, b(X) \in k$. For each $Z \in S$, we have $\Sigma\left(\lambda_{Z}\right)=\lambda_{\Sigma(Z)}$. Applying the naturality of $\lambda$ to the isomorphism $t_{Z}$ in (2.1) and using (2.2), we infer that $b(Z)=b\left(Z^{\prime}\right)$. Then the function $b$ is constant on each $\Sigma$-orbit of $S$. In other words, $\lambda=c+$ $\sum_{[X] \in S / \Sigma} b(X) \delta([X])$. This proves $(1)$.

For (2), we have an invertible element $\lambda \in Z(\mathcal{T})$ satisfying $\omega=\Sigma(\lambda)$; moreover, by Proposition 2.5(2), we may assume that

$$
\lambda=1+\sum_{X \in S} b(X) \delta(X)
$$


for $b(X) \in k$. For each $X \in S$, we choose a scalar $\phi(X)$ such that $\phi(X)-\phi\left(X^{\prime}\right)=$ $b(X)$, where $X^{\prime} \in S$ is uniquely determined by the isomorphism (2.1). This choice of the function $\phi(-)$ is possible, since the $\Sigma$-orbit $[X]$ is infinite.

Set $\eta=1+\sum_{X \in S} \phi(X) \delta(X) \in Z(\mathcal{T})$, which is invertible. We claim that $\eta:\left(\operatorname{Id}_{\mathcal{T}}, \omega\right) \rightarrow\left(\operatorname{Id}_{\mathcal{T}}, \operatorname{Id}_{\Sigma}\right)$ is the required isomorphism.

To prove that $\eta$ is a natural transformation between triangle functors, it suffices to verify $\eta_{\Sigma(U)} \circ \omega_{U}=\Sigma\left(\eta_{U}\right)$ for each $U \in \operatorname{ind} \mathcal{T}$. Recall that $\omega=\Sigma(\lambda)$ and that the value of $\delta(X)$ on $X$ is $\Delta_{X}$ for each $X \in S$. The left square in the following commutative diagram

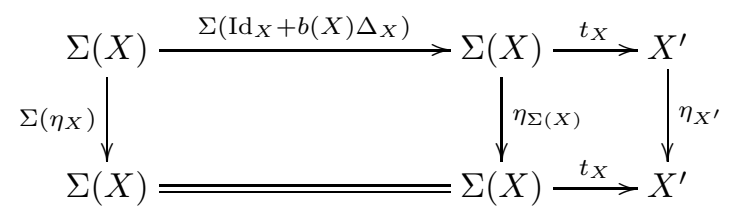

proves $\eta_{\Sigma(X)} \circ \omega_{X}=\Sigma\left(\eta_{X}\right)$. Here, the commutativity of the outer diagram follows from the choice of the function $\phi(-)$, since by (2.2) we have

$$
\left(t_{X}\right)^{-1} \circ \eta_{X^{\prime}} \circ t_{X}=\Sigma\left(\operatorname{Id}_{X}+\phi\left(X^{\prime}\right) \Delta_{X}\right) \text {. }
$$

The identity $\eta_{\Sigma(Y)} \circ \omega_{Y}=\Sigma\left(\eta_{Y}\right)$ is clear for each $Y \in S^{\prime}$. This completes the proof of the claim.

\section{The MAIN RESUlts}

In this section, we first recall from 8 , the notion of a $\mathbf{K}$-standard category. The main techical result claims that the category of projective modules over a certain Nakayama algebra is $\mathbf{K}$-standard; see Theorem 3.5. Then using the classification result in [4, we deduce that every derived equivalence between derived-discrete algebras of finite global dimension is standard; see Theorem 3.6 .

Let $k$ be a field and $\mathcal{A}$ be a $k$-linear additive category. We denote by $\mathbf{K}^{b}(\mathcal{A})$ the bounded homotopy category. A bounded complex $X=\left(X^{n}, d_{X}^{n}\right)_{n \in \mathbb{Z}}$ is visualized as

$$
\cdots \longrightarrow X^{n} \stackrel{d_{X}^{n}}{\longrightarrow} X^{n+1} \stackrel{d_{X}^{n+1}}{\longrightarrow} X^{n+2} \longrightarrow \cdots,
$$

where $X^{n}=0$ for $|n| \gg 0$. The support of $X$, denoted by $\operatorname{supp}(X)$, is the interval $[m, n]$ in $\mathbb{Z}$, where $m$ is the smallest integer with $X^{m} \neq 0$, and $n$ the largest integer with $X^{n} \neq 0$. Two homotopic complexes may have different supports.

The suspension functor $\Sigma$ sends $X$ to $\Sigma(X)$, which is given by $\Sigma(X)^{n}=X^{n+1}$ and $d_{\Sigma(X)}^{n}=-d_{X}^{n+1}$. We identify $\mathcal{A}$ as the full subcategory of $\mathbf{K}^{b}(\mathcal{A})$ consisting of stalk complexes concentrated on degree zero. Therefore, for each integer $n$, an object $M \in \mathcal{A}$ yields a stalk complex $\Sigma^{n}(M)$ concentrated on degree $-n$. These complexes form a full subcategory $\Sigma^{n}(\mathcal{A})$, where $\mathcal{A}=\Sigma^{0}(\mathcal{A})$.

We have the following $k$-algebra homomorphism

$$
\text { res: } Z_{\Delta}\left(\mathbf{K}^{b}(\mathcal{A})\right) \longrightarrow Z(\mathcal{A})
$$

sending $\lambda$ to $\left.\lambda\right|_{\mathcal{A}}$, the restriction of $\lambda$ to $\mathcal{A}$. This homomorphism has a right inverse, which sends $\mu \in Z(\mathcal{A})$ to $\mathbf{K}^{b}(\mu)$, the natural extension of $\mu$ on complexes.

The following notions are introduced in [8, Sections 3 and 4]. By [8, Lemma 4.2], the current notion of a $\mathbf{K}$-standard category is equivalent to the original one.

Definition 3.1. (1) A $k$-linear triangle endofunctor $(F, \omega)$ on $\mathbf{K}^{b}(\mathcal{A})$ is a pseudoidentity, provided that $F(X)=X$ for each complex $X$, and for each integer $n$ the restriction $\left.F\right|_{\Sigma^{n}(\mathcal{A})}: \Sigma^{n}(\mathcal{A}) \rightarrow \Sigma^{n}(\mathcal{A})$ equals the identity functor.

(2) The $k$-linear additive category $\mathcal{A}$ is $\mathbf{K}$-standard (over $k$ ), provided that each 
pseudo-identity functor is isomorphic to $\left(\operatorname{Id}_{\mathbf{K}^{b}(\mathcal{A})}, \operatorname{Id}_{\Sigma}\right)$, the genuine identity functor; it is strongly $\mathbf{K}$-standard, if in addition the homomorphism (3.1) is injective.

We say that a pseudo-identity $(F, \omega)$ on $\mathbf{K}^{b}(\mathcal{A})$ is normalized, provided that $\omega_{X}=\Sigma\left(\operatorname{Id}_{X}\right)$ for each stalk complex $X$. Here, by a stalk complex, we mean a complex of the form $\Sigma^{n}(M)$ for some integer $n$ and $M \in \mathcal{A}$. According to the following observation, up to isomorphism, we may always assume that any pseudoidentity is normalized.

Lemma 3.2. Let $(F, \omega)$ be a pseudo-identity on $\mathbf{K}^{b}(\mathcal{A})$. Then there exists a normalized pseudo-identity $\left(F^{\prime}, \omega^{\prime}\right)$ with an isomorphism $\delta:(F, \omega) \rightarrow\left(F^{\prime}, \omega^{\prime}\right)$.

Proof. We observe that, for each $n \in \mathbb{Z}$, there is a unique invertible element $\lambda_{n} \in$ $Z(\mathcal{A})$ such that for each object $M \in \mathcal{A}$, the connecting isomorphism

$$
\omega_{\Sigma^{n}(M)}: F \Sigma\left(\Sigma^{n} M\right)=\Sigma^{n+1}(M) \longrightarrow \Sigma^{n+1}(M)=\Sigma F\left(\Sigma^{n} M\right)
$$

equals $\Sigma^{n+1}\left(\left(\lambda_{n}\right)_{M}\right)$. Take a sequence $\left\{a_{n}\right\}_{n \in \mathbb{Z}}$ of invertible elements in $Z(\mathcal{A})$ such that $a_{0}=1$ and $\lambda_{n}=a_{n}^{-1} a_{n+1}$ for each integer $n$.

For each complex $X$, we take an isomorphism $\delta_{X}: F(X)=X \rightarrow X=F^{\prime}(X)$ such that $\delta_{\Sigma^{n}(M)}=\Sigma^{n}\left(\left(a_{n}\right)_{M}\right)$ for each integer $n$ and $M \in \mathcal{A}$. We use $\delta_{X}$ 's as the adjusting isomorphisms to obtain the required isomorphism; see Lemma 2.1] By the construction of $\left(F^{\prime}, \omega^{\prime}\right)$ in the proof, we infer that $\left(F^{\prime}, \omega^{\prime}\right)$ is a normalized pseudo-identity. Here, we recall that $\omega^{\prime}=(\Sigma \delta) \circ \omega \circ(\delta \Sigma)^{-1}$, from which and the choice of the sequence $\left\{a_{n}\right\}_{n \in \mathbb{Z}}$ we deduce that $\omega_{\Sigma^{n}(M)}^{\prime}$ is the identity.

Let $A$ be a finite dimensional $k$-algebra. We denote by $A$-mod the abelian category of finite dimensional left $A$-modules, and by $A$-proj the full subcategory formed by projective $A$-modules. Denote by $\mathbf{D}^{b}(A$-mod) the bounded derived category.

Two algebras $A$ and $B$ are derived equivalent provided that there is a $k$-linear triangle equivalence $(F, \omega): \mathbf{D}^{b}(A$-mod $) \rightarrow \mathbf{D}^{b}(B$-mod $)$, called a derived equivalence. For any $B$ - $A$-bimodule, we always require that $k$ acts centrally. A bounded complex $X$ of $B$-A-bimodules is a called two-sided tilting complex provided that the derived tensor functor $X \otimes_{A}^{\mathbb{L}}-: \mathbf{D}^{b}(A$-mod $) \rightarrow \mathbf{D}^{b}(B$-mod $)$ is an equivalence. A derived equivalence $(F, \omega)$ is standard, if it is isomorphic to $X \otimes_{A}^{\mathbb{L}}-$, as a triangle functor, for some two-sided tilting complex $X$. It is an open question in [14] whether all derived equivalences are standard. For details on derived equivalences, we refer to [16.

The following result is the main motivation to study the $\mathbf{K}$-standardness, which is obtained by combining [8, Theorems 6.1 and 5.10].

Proposition 3.3. Let $A$ and $B$ be two finite dimensional $k$-algebras such that $A$-proj is $\mathbf{K}$-standard. Then any derived equivalence $\mathbf{D}^{b}(A$-mod $) \rightarrow \mathbf{D}^{b}(B$-mod $)$ is standard.

The following observation will be needed.

Lemma 3.4. Let $A$ and $B$ be two finite dimensional $k$-algebras of finite global dimension, which are derived equivalent. Then $A$-proj is $\mathbf{K}$-standard if and only if so is $B$-proj.

Proof. By [8, Section 6], $A$-proj is $\mathbf{K}$-standard if and only if the module category $A$-mod is $\mathbf{D}$-standard in the sense of [8, Definition 5.1]. Then the desired equivalence follows from [8, Lemma 5.12]. 
Let $r \geq 1$ and $N \geq r$. Let $A=A(r, N)$ be the finite dimensional algebra given by the following cyclic quiver

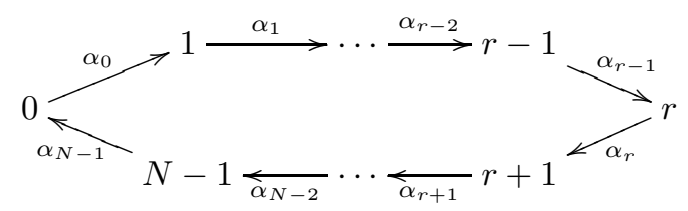

with $r$ relations $\left\{\alpha_{0} \alpha_{N-1}, \alpha_{1} \alpha_{0}, \cdots, \alpha_{r-1} \alpha_{r-2}\right\}$. Here, we write the concatenation of arrows from right to left. Then $A$ is a Nakayama algebra. For each vertex $i$, we denote by $e_{i}$ the corresponding primitive idempotent.

We assume that $r<N$. Then the global dimension of $A$ is $r+1$. For $0 \leq i \leq r-1$, we denote by $P_{i}=A e_{i}$ the indecomposable projective module corresponding to $i$. Similarly, we have $Q_{a}=A e_{a}$ for $r \leq a<N$. We observe that $\left\{P_{0}, P_{1}, \cdots, P_{r-1}\right\}$ is a complete set of representatives of isoclasses of indecomposable projective-injective A-modules.

The following convention of unnamed arrows will be used later.

Convention $(\dagger) \quad$ For $1 \leq i \leq r-1$, we denote by the unnamed arrow $P_{i} \rightarrow P_{i-1}$ the unique $A$-module homomorphism sending $e_{i}$ to $\alpha_{i-1}$. The unnamed arrow $P_{0} \rightarrow$ $P_{r-1}$ sends $e_{0}$ to the path $\alpha_{N-1} \cdots \alpha_{r} \alpha_{r-1}$. Indeed, we will understand the index $i$ in the cyclic group $\mathbb{Z} / r \mathbb{Z}$. For $r \leq a<N$, we denote by the unnamed arrows $Q_{a} \rightarrow$ $P_{r-1}$ and $P_{0} \rightarrow Q_{a}$ the unique homomorphisms sending $e_{a}$ to $\alpha_{a-1} \cdots \alpha_{r} \alpha_{r-1}$, and $e_{0}$ to $\alpha_{N-1} \cdots \alpha_{a+1} \alpha_{a}$, respectively. For $r \leq b<a<N$, the unnamed arrow $Q_{a} \rightarrow Q_{b}$ sends $e_{a}$ to the unique path going from $b$ to $a$.

The main result of this paper is as follows.

Theorem 3.5. Let $1 \leq r \leq N$ and $A=A(r, N)$ be the above algebra. Then the following statements hold.

(1) If $r=1$, then the category $A$-proj is $\mathbf{K}$-standard, but not strongly $\mathbf{K}$ standard.

(2) If $r>1$, then the category $A$-proj is strongly $\mathbf{K}$-standard.

Consequently, any derived equivalence $\mathbf{D}^{b}(A(r, N)$-mod $) \rightarrow \mathbf{D}^{b}(B$-mod $)$ is standard.

For the proof, the last statement follows from Proposition 3.3. The case $r=N$ is treated in [8, Section 7]. Assume that $r<N$. We will prove (1) in Section 4 and (2) in Section 5.

For the rest of this section, we assume that the base field $k$ is algebraically closed. We will apply Theorem 3.5 to derived-discrete algebras of finite global dimension.

Recall from 15 that a finite dimensional $k$-algebra $A$ is derived-discrete, provided that for each vector $\mathbf{n}=\left(n_{i}\right)_{i \in \mathbb{Z}}$ of natural numbers there are only finitely many isomorphism classes of indecomposable objects in $\mathbf{D}^{b}(A$-mod) with cohomology dimension vector $\mathbf{n}$. Here, for a complex $X$ of $A$-modules, its cohomology dimension vector is given by $\left(\operatorname{dim} H^{i}(X)\right)_{i \in \mathbb{Z}}$. By [15, Proposition 1.1], deriveddiscrete algebras are closed under derived equivalences.

By the classification result [4] of derived-discrete algebras up to derived equivalence, we have the following result.

Theorem 3.6. Let $k$ be an algebraically closed field, and $A$ be a derived-discrete $k$-algebra of finite global dimension. Then the category A-proj is $\mathbf{K}$-standard. Consequently, any derived equivalence between derived-discrete algebras of finite global dimension is standard. 
Proof. The second statement follows from Proposition 3.3. Without loss of generality, we may assume that the algebra $A$ is connected.

For the first statement, it suffices to claim that $A$ is derived equivalent to a triangular algebra $C$, or the algebra $A(r, N)$ for $1 \leq r<N$. By [8, Proposition 5.13] and Theorem 3.5. we infer that for both $C$ and $A(r, N)$, their categories of projective modules are $\mathbf{K}$-standard. Then we are done by Lemma 3.4.

For the claim, we recall from [15, Theorem 2.1] and 4, Proposition 2.3] that $A$ is either derived equivalent to the path algebra of a Dynkin quiver, or to the algebra $A(r, N, m)$ given by the following quiver

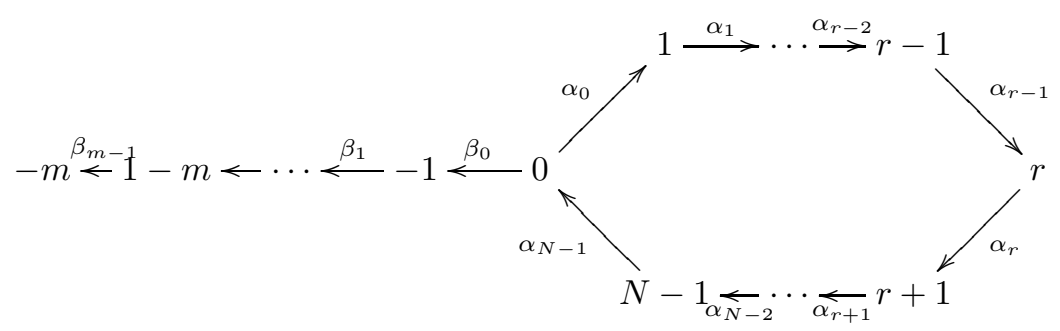

with $r$ relations $\left\{\alpha_{0} \alpha_{N-1}, \alpha_{1} \alpha_{0}, \cdots, \alpha_{r-1} \alpha_{r-2}\right\}$, where $1 \leq r<N$ and $m \geq 0$. We observe that $A(r, N, 0)=A(r, N)$. If $m>0$, the algebra $A(r, N, m)$ is derived equivalent to a triangular algebra; see [4, Proof $2.4(\mathrm{~h})]$. This proves the claim.

Remark 3.7. (1) Recall from [10, 5] that the group of standard autoequivalences on $\mathbf{D}^{b}(A$-mod) is explicitly calculated, where $A$ is a derived-discrete algebra of finite global dimension. Theorem 3.6 implies that this group coincides with the whole group of (triangle) autoequivalences on $\mathbf{D}^{b}(A$-mod).

(2) It is natural to expect that Theorem 3.6 also holds for a derived-discrete algebra of infinite global dimension. By [4, Proposition 2.3], it suffices to prove that $A(r, r, m)$-proj is $\mathbf{K}$-standard for $m \geq 1$. However, the argument in this paper does not apply to the algebras $A(r, r, m)$. It seems that the most difficult case is the algebra $A(1,1, m)$.

\section{THE CASE $r=1$}

In this section, we prove Theorem 3.5 for the case $r=1$ and $N>1$.

Let $A=A(1, N), \mathcal{A}=A$-proj and $\mathcal{T}=\mathbf{K}^{b}(\mathcal{A})$. We will use Convention ( $\dagger$ ) in Section 3. Consider the following objects in $\mathcal{T}$ :

$$
\begin{aligned}
X_{m, n} & =\cdots \rightarrow 0 \rightarrow P_{0} \rightarrow P_{0} \rightarrow \cdots \rightarrow P_{0} \rightarrow P_{0} \rightarrow 0 \rightarrow \cdots \\
L_{m, n, a} & =\cdots \rightarrow 0 \rightarrow Q_{a} \rightarrow P_{0} \rightarrow P_{0} \rightarrow \cdots \rightarrow P_{0} \rightarrow P_{0} \rightarrow 0 \rightarrow \cdots \\
R_{m, n, b} & =\cdots \rightarrow 0 \rightarrow P_{0} \rightarrow P_{0} \rightarrow \cdots \rightarrow P_{0} \rightarrow P_{0} \rightarrow Q_{b} \rightarrow 0 \rightarrow \cdots \\
B_{m, n, a, b} & =\cdots \rightarrow 0 \rightarrow Q_{a} \rightarrow P_{0} \rightarrow P_{0} \rightarrow \cdots \rightarrow P_{0} \rightarrow P_{0} \rightarrow Q_{b} \rightarrow 0 \rightarrow \cdots \\
Z_{m, a, b} & =\cdots \rightarrow 0 \rightarrow Q_{a} \rightarrow Q_{b} \rightarrow 0 \rightarrow \cdots .
\end{aligned}
$$

Some comments on the lower indices are necessary. The supports of $X_{m, n}, L_{m, n, a}$ and $R_{m, n, b}$ are $[m, n]$ for $m \leq n$, where $a, b$ run from 1 to $N-1$. We observe that $X_{m, m}=\Sigma^{-m}\left(P_{0}\right)$ and $L_{m, m, a}=R_{m, m, a}=\Sigma^{-m}\left(Q_{a}\right)$. For $m \leq n-2$ and $1 \leq a, b<N$, the complex $B_{m, n, a, b}$ is defined, whose support is $[m, n]$. The complex $Z_{m, a, b}$ is supported in $[m, m+1]$, where $1 \leq b<a<N$. In particular, the complex $Z_{m, a, b}$ is not defined for the case $N=2$.

Lemma 4.1. Keep the above notation. Then

$$
\Lambda=\left\{X_{m, n}, L_{m, n, a}, R_{m, n, b}, B_{m, n, a, b}, Z_{m, a, b}\right\}
$$

is a complete set of representatives of isoclasses of indecomposable objects in $\mathcal{T}$. 
Proof. By [2, Theorem 3], the isoclasses of indecomposable objects in $\mathcal{T}$, up to suspensions, are in a bijection to the generalized strings in $A$. For $1 \leq a, b<N$ and $p \geq 0$, we consider the following generalized string

$$
w_{a, b}^{p}:=w_{0} w_{1} \cdots w_{p} w_{p+1}=\left(\alpha_{b} \cdots \alpha_{1} \alpha_{0}\right)\left(\alpha_{N-1} \cdots \alpha_{1} \alpha_{0}\right)^{p}\left(\alpha_{N-1} \cdots \alpha_{a+1} \alpha_{a}\right),
$$

where $w_{0}=\alpha_{b} \cdots \alpha_{1} \alpha_{0}, w_{1}=w_{2}=\cdots=w_{p}=\alpha_{N-1} \cdots \alpha_{1} \alpha_{0}$ and $w_{p+1}=$ $\alpha_{N-1} \cdots \alpha_{a+1} \alpha_{a}$ are nonzero paths in $A$. It is clear that any generalized string $w$ is a generalized substring of $w_{a, b}^{p}$, that is, of the form $w_{i} w_{i+1} \cdots w_{j}$ for some $0 \leq i \leq$ $j \leq p+1$. Then the indecomposable object $P_{w}$ corresponding to $w$ is isomorphic to an element in $\Lambda$; compare [2, Definition 2]. For example, up to suspensions, the complex $B_{m, n, a, b}$ corresponds to the generalized string $w_{a, b}^{n-m-2}$.

Remark 4.2. We compute the endomorphism algebras of objects in $\Lambda$. We have $\operatorname{End}_{\mathcal{T}}\left(X_{m, n}\right)=k \operatorname{Id}_{X_{m, n}} \oplus k \Delta_{m, n}$, where $\Delta_{m, n}$ is of the following form.

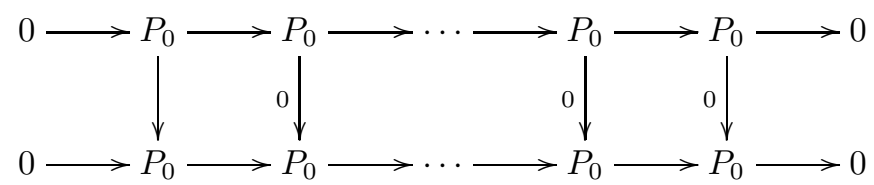

Here, the unique nonzero vertical map is the unnamed arrow $P_{0} \rightarrow P_{0}$, sending $e_{0}$ to $\alpha_{N-1} \cdots \alpha_{1} \alpha_{0}$. We observe that the endomorphism $\Delta_{m, n}$ is almost-vanishing, since $\nu\left(X_{m, n}\right) \simeq X_{m, n}$ with $\nu$ the Nakayama functor; see [9, I.4.6]. For other objects $Y$ in $\Lambda$, we have $\operatorname{End}_{\mathcal{T}}(Y)=k \operatorname{Id}_{Y}$.

In particular, the category $\mathcal{T}$ satisfies the condition (A2) in Section 2, where $S$ is the subset of $\Lambda=\operatorname{ind} \mathcal{T}$ consisting of the complexes $X_{m, n}$. Moreover, the isomorphism (2.1) for $X_{m, n}$ is given by

$$
t_{m, n}: \Sigma\left(X_{m, n}\right) \longrightarrow X_{m-1, n-1},
$$

where its $p$-the component $t_{m, n}^{p}$ is by multiplying $(-1)^{p}$. Then we have (2.2) for $X_{m, n}$, that is,

$$
\Delta_{m-1, n-1}=t_{m, n} \circ \Sigma\left(\Delta_{m, n}\right) \circ t_{m, n}^{-1}
$$

In what follows, we will describe morphisms between the objects in $\Lambda$. One might find these morphisms in [1, where a combinatorial description of morphisms between the indecomposables in the bounded derived category of gentle algebras is available. We try to make this exposition self-contained, and use the notation for our convenience. We divide the morphisms between objects in $\Lambda$ into four types: inclusions, projections, connections and morphisms of the mixed type.

The first type is induced by the obvious inclusion: $i_{m, n}: X_{m, n} \rightarrow X_{m-1, n}$, $j_{m, n, a}: X_{m, n} \rightarrow L_{m-1, n, a}, i_{m, n, b}^{\prime}: R_{m, n, b} \rightarrow R_{m-1, n, b}$ for $m \leq n$ and $1 \leq a, b<N$; $\iota_{m, n, a, b}: R_{m, n, b} \rightarrow B_{m-1, n, a, b}$ for $m<n$ and $1 \leq a, b<N ; \xi_{m, a, b}: L_{m, m, b} \rightarrow$ $Z_{m-1, a, b}$ for each integer $m$ and $1 \leq b<a<N$. We will call any composition of these morphisms an inclusion, which will be denoted by "inc".

The second type is induced by the obvious projection: $\pi_{m, n}: X_{m, n} \rightarrow X_{m, n-1}$, $\pi_{m, n, a}^{\prime}: L_{m, n, a} \rightarrow L_{m, n-1, a}$ and $p_{m, n, b}: R_{m, n, b} \rightarrow X_{m, n-1}$ for $m<n$ and $1 \leq a, b<$ $N ; q_{m, n, a, b}: B_{m, n, a, b} \rightarrow L_{m, n-1, a}$ for $m \leq n-2$ and $1 \leq a, b<N ; \zeta_{m, a, b}: Z_{m, a, b} \rightarrow$ $L_{m, m, a}$ for each integer $m$ and $1 \leq b<a<N$. We will call any composition of these morphisms a projection, which will be denoted by "pr". 
For the third type, we denote by $c_{l, m, n, a, b}: L_{l, m, a} \rightarrow R_{m, n, b}$, for $l \leq m \leq n$ and $1 \leq a, b<N$, the following morphism

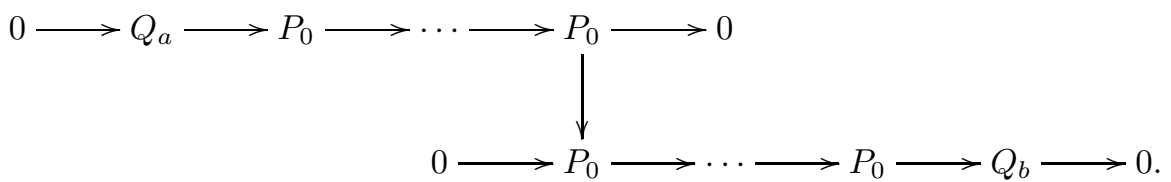

Here, as usual, the unique nonzero vertical map $P_{0} \rightarrow P_{0}$ sends $e_{0}$ to the path $\alpha_{N-1} \cdots \alpha_{1} \alpha_{0}$. If $l=m<n$, the vertical map is given by the unnamed arrow $Q_{a} \rightarrow P_{0}$; if $l<m=n$, it is given by $P_{0} \rightarrow Q_{b}$. If $l=m=n$, we have to assume that $b<a$, in which case we have $c_{l, l, l, a, b}=\Sigma^{-l}(\phi)$, where $\phi: Q_{a} \rightarrow Q_{b}$ is the unnamed arrow. We will call any morphism of the form

$$
\operatorname{pr} \circ c_{l, m, n, a, b} \circ \text { inc }
$$

a connection. For example, the following morphism $c_{l, m, n}: X_{l, m} \rightarrow X_{m, n}$

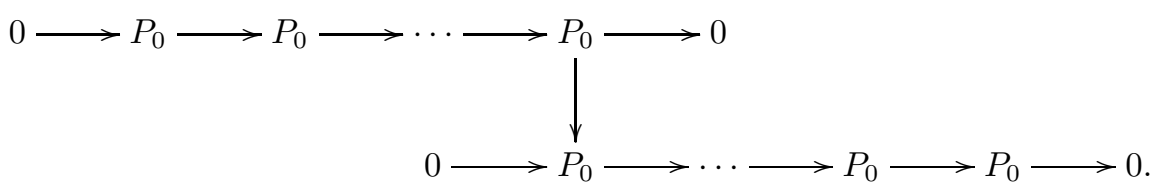

is a connection for $l \leq m \leq n$. We observe that the composition

$$
X_{m, n} \stackrel{\mathrm{pr}}{\longrightarrow} X_{m, m} \stackrel{c_{m, m, n}}{\longrightarrow} X_{m, n}
$$

equals the almost-vanishing endomorphism $\Delta_{m, n}$ in Remark 4.2

The fourth type, called the mixed type, is divided into 11 classes of morphisms. They are listed as follows. Here, "mx" stands for the word "mixed".

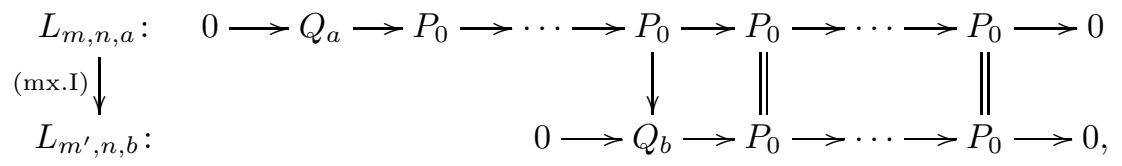

for $m<m^{\prime}<n$ and $1 \leq a, b<N$;

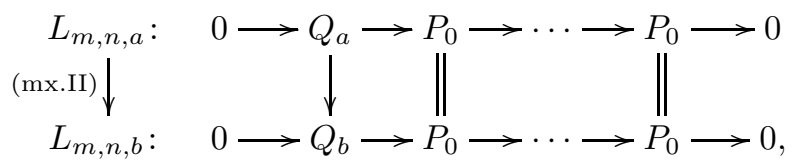

for $m<n$ and $1 \leq b<a<N$;

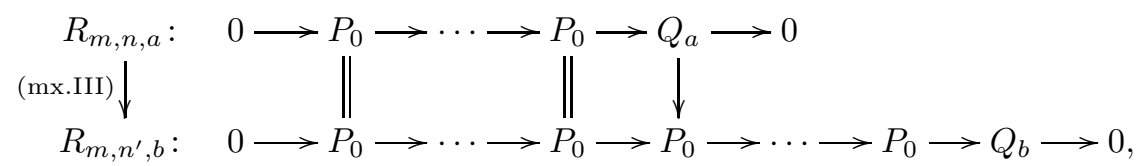

for $m<n<n^{\prime}$ and $1 \leq a, b<N$;

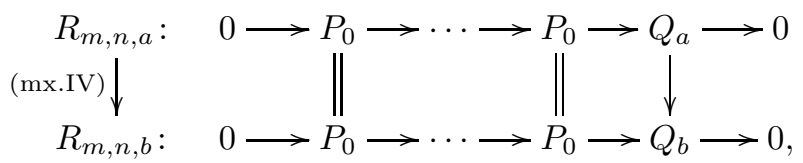


for $m<n$ and $1 \leq b<a<N$;

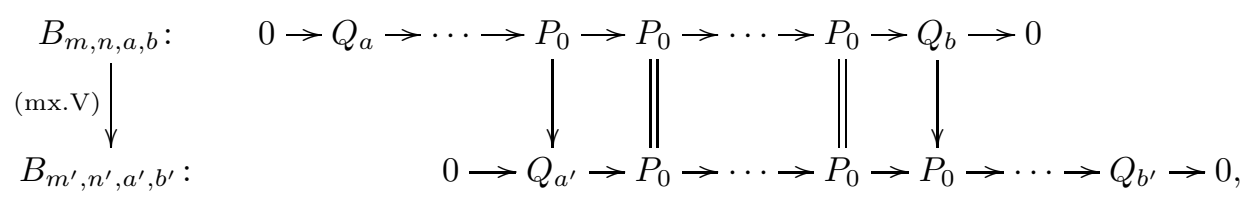

for $m<m^{\prime}<n<n^{\prime}$ and $1 \leq a, b, a^{\prime}, b^{\prime}<N$;

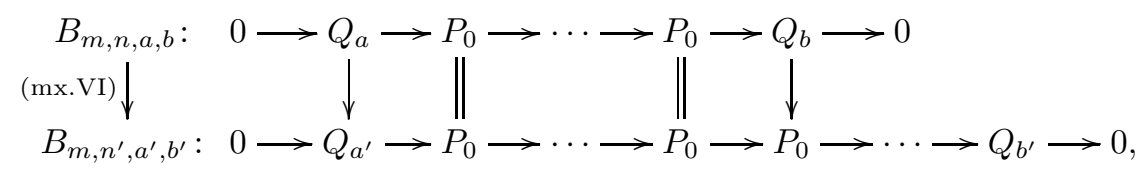

for $m+2 \leq n<n^{\prime}$ and $1 \leq a, b, a^{\prime}, b^{\prime}<N$ with $a^{\prime} \leq a$, where for the case $a=a^{\prime}$ the unnamed arrow $Q_{a} \rightarrow \bar{Q}_{a^{\prime}}$ is the identity map; dually, we have

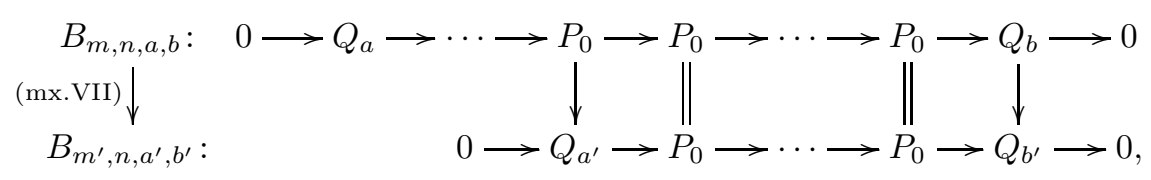

for $m<m^{\prime} \leq n-2$ and $1 \leq a, b, a^{\prime}, b^{\prime}<N$ with $b^{\prime} \leq b$, where for the case $b=b^{\prime}$ the unnamed arrow $Q_{b} \rightarrow Q_{b^{\prime}}$ is the identity map;

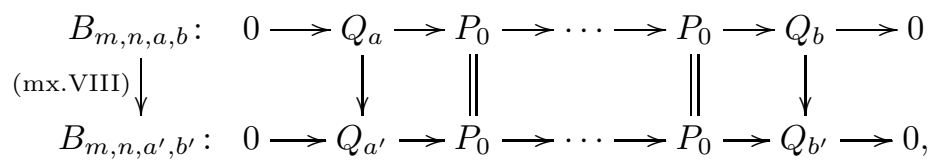

for $m \leq n-2, a^{\prime} \leq a$ and $b^{\prime} \leq b$;

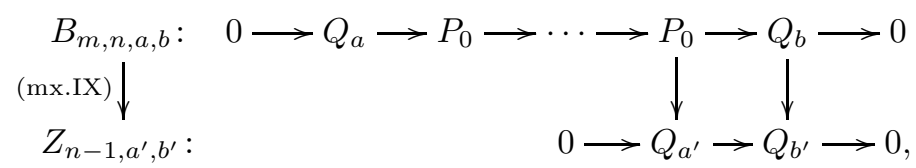

for $m \leq n-2$ and $1 \leq b^{\prime} \leq b<a^{\prime}<N$;

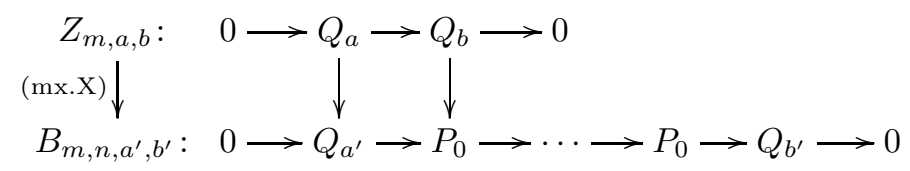

for $m \leq n-2$ and $1 \leq b<a^{\prime} \leq a<N$;

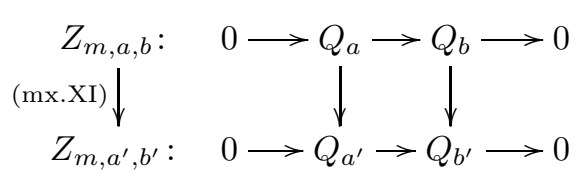

for each integer $m$ and $1 \leq b^{\prime} \leq b<a^{\prime} \leq a<N$.

We mention that a morphism of class $(\mathrm{mx} . \mathrm{V})$ is zero provided that $n=m^{\prime}+1$ and $a^{\prime} \leq b$. The morphisms of classes (mx.VI), (mx.VII) and (mx.VIII) might be viewed as degenerate cases of the ones of class $(\mathrm{mx} . \mathrm{V})$, by requiring that $n=n^{\prime}$ or $m=m^{\prime}$.

The first statement of the following result might be deduced from [1, while the second is essentially due to [5, Proposition 6.2].

Proposition 4.3. Keep the notation as above. Then the following statements hold. 
(1) The above morphisms

$$
\begin{aligned}
& \left\{i_{m, n}, j_{m, n, a}, i_{m, n, b}^{\prime}, \iota_{m, n, a, b}, \xi_{m, a, b}\right\} \cup\left\{\pi_{m, n}, \pi_{m, n, a}^{\prime}, p_{m, n, b}, q_{m, n, a, b}, \zeta_{m, a, b}\right\} \\
& \cup\left\{c_{l, m, n, a, b}\right\} \cup\{(\mathrm{mx} . \mathrm{I})-(\mathrm{mx} . \mathrm{XI})\} \\
& \quad \text { form a spanning set of } \mathcal{T} . \\
& \text { (2) For two objects } X, Y \in \Lambda, \text { we have } \operatorname{dim}_{\operatorname{Hom}} \operatorname{Hom}_{\mathcal{T}}(X, Y) \leq 2 ; \text { moreover, the } \\
& \quad \text { equality holds if and only if } X=Y=X_{m, n} .
\end{aligned}
$$

We make some preparations for the proof. The following terminology will be convenient. For two objects $X, Y \in \Lambda$ with $\operatorname{supp}(X)=[m, n]$ and $\operatorname{supp}(Y)=$ $[p, q]$, a nonzero morphism $f: X \rightarrow Y$ is said to be crossing provided that for any morphism $g: X \rightarrow Y$, which is homotopic to $f$, satisfies $g^{n} \neq 0$ and $g^{p} \neq 0$. It follows that the pair $([m, n],[p, q])$ of intervals is necessarily crossing, meaning that $m \leq p \leq n \leq q$. We call the positive integer $n-p+1$ the width of $f$.

Lemma 4.4. For any given objects $X, Y \in \Lambda$, there exist two objects $X^{\prime}$ and $Y^{\prime}$ in $\Lambda$ with an inclusion inc: $Y^{\prime} \rightarrow Y$ and a projection $\mathrm{pr}: X \rightarrow X^{\prime}$ such that the pair $\left(\operatorname{supp}\left(X^{\prime}\right), \operatorname{supp}\left(Y^{\prime}\right)\right)$ is crossing and that the map is surjective

$$
\operatorname{Hom}_{\mathcal{T}}\left(X^{\prime}, Y^{\prime}\right) \longrightarrow \operatorname{Hom}_{\mathcal{T}}(X, Y), \quad f \mapsto \operatorname{inc} \circ f \circ \mathrm{pr} .
$$

Proof. We may assume that $\operatorname{Hom}_{\mathcal{T}}(X, Y) \neq 0$ and that $(\operatorname{supp}(X), \operatorname{supp}(Y))$ is not crossing. Assume that $\operatorname{supp}(X)=[m, n]$ and $\operatorname{supp}(Y)=[p, q]$. Then there are three cases: $p<m \leq n<q$, or $m<p \leq q<n$, or $p<m \leq q<n$. In each case, we truncate $X$ or $Y$ to obtain the desired $X^{\prime}$ or $Y^{\prime}$.

Lemma 4.5. Assume that $X, Y \in \Lambda$ satisfy that the pair $(\operatorname{supp}(X), \operatorname{supp}(Y))$ is crossing. Let $f: X \rightarrow Y$ be a nonzero morphism in $\mathcal{T}$. Then there exist two objects $X^{\prime}$ and $Y^{\prime}$ in $\Lambda$ with an inclusion inc: $Y^{\prime} \rightarrow Y$, a projection pr: $X \rightarrow X^{\prime}$ and a crossing morphism $f^{\prime}: X^{\prime} \rightarrow Y^{\prime}$ satisfying $f=$ inc $\circ f^{\prime} \circ \operatorname{pr}$ in $\mathcal{T}$.

Proof. If $f$ is already crossing, we take $X^{\prime}=X$ and $Y=Y^{\prime}$. Otherwise, we assume that $\operatorname{supp}(X)=[m, n]$ and $\operatorname{supp}(Y)=[p, q]$. Up to homotopy, we have $f^{n}=0$ or $f^{p}=0$. In the first case, we truncate $X$ to obtain $X^{\prime}$; in the latter case, we truncate $Y$. Then we are done by induction on the width.

We now sketch a proof for Proposition 4.3

Proof. For (1), we apply the above two lemmas. It suffices to prove the following claim: up to nonzero scalars, any crossing morphism $f: X \rightarrow Y$ is a composition of the listed morphisms. If the width of $f$ is one, then up to nonzero scalars, $f$ is a connection, and then we are done. We may assume that the width of $f$ is at least two. We have to analyse such morphisms between objects in $\Lambda$ case by case; since $\Lambda$ is divided into 5 classes, we have here 25 cases to verify the claim. But each case is easy to verify. We omit the details.

For $(2)$, we apply Lemma 4.4. We may assume that the pair $(\operatorname{supp}(X), \operatorname{supp}(Y))$ is crossing. It suffices to claim $\operatorname{dim} \operatorname{Hom}_{\mathcal{T}}(X, Y) \leq 2$, and the equality holds if and only if $X=Y=X_{m, n}$. The claim implies the desired results, since $\operatorname{End}_{\mathcal{T}}\left(X_{m, n}\right)=$ $k \operatorname{Id}_{X_{n, m}} \oplus k \Delta_{m, n}$ with $\Delta_{m, n}$ an almost-vanishing morphism. The proof of the claim is done by computing the Hom-spaces $\operatorname{Hom}_{\mathcal{T}}(X, Y)$ for the 25 cases, under the assumption that $(\operatorname{supp}(X), \operatorname{supp}(Y))$ is crossing.

We are in a position to prove Theorem 3.5 for the case $r=1$. We will often use Lemma 2.1 to adjust the pseudo-identities on $\mathcal{T}$.

In what follows, if we have $F(f)=f$ for an endofunctor $F$ on $\mathcal{T}$ and a morphism $f$ in $\mathcal{T}$, then we say that $F$ acts trivially on $f$, or acts on $f$ by the identity. 
Proof. Let $(F, \omega)$ be a pseudo-identity on $\mathcal{T}$. We observe first that $F$ acts on the morphisms in Proposition 4.3(1) by nonzero scalars.

We will adjust $F$ by nonzero scalars to obtain a new pseudo-identity $(\bar{F}, \bar{\omega})$ such that $\bar{F}$ acts trivially on the spanning set in Proposition $4.3(1)$. By Lemma 2.2, as a triangle functor, $(\bar{F}, \bar{\omega})$ is isomorphic to $\left(\operatorname{Id}_{\mathcal{T}}, \omega^{\prime}\right)$ for some natural automorphism $\omega^{\prime}$ on $\Sigma$.

By Remark 4.2, Proposition 2.6 applies to $\mathcal{T}$. By Proposition 2.6 (2), the triangle functor $\left(\operatorname{Id}_{\mathcal{T}}, \omega^{\prime}\right)$ is isomorphic to the identity functor. This proves that, as a triangle functor, the given $(F, \omega)$ is isomorphic to the identity functor. Then $\mathcal{A}$ is K-standard. In view of Proposition 2.6 (1), the homomorphism (3.1) is not injective, since the $\Sigma$-orbit set $S / \Sigma$ is infinite. This also follows from [3. Proposition 4.5]. Here, we use the fact that the center $Z(\mathcal{A})$ is isomorphic to the center of the algebra $A$, which is isomorphic to the algebra of dual numbers. Then the category $\mathcal{A}$ is not strongly K-standard. We are done.

In what follows, we will adjust the given pseudo-identity $(F, \omega)$ in Step 1. In Step $2-4$, we show that the adjusted pseudo-identity acts trivially on the spanning set in Proposition 4.3(1). By Lemma 3.2 we may assume that the given pseudo-identity $(F, \omega)$ is normalized.

Step 1 For each $X \in \Lambda$ with $\operatorname{supp}(X)=[m, n]$, we denote by $\operatorname{inc}_{X}: \Sigma^{-n}\left(X^{n}\right) \rightarrow$ $X$ the obvious inclusion. If $m=n$, inc ${ }_{X}$ is the identity map. We may assume that $F\left(\right.$ inc $\left._{X}\right)=\phi(X)$ inc $_{X}$ with $\phi(X)$ a nonzero scalar.

For each complex $Y$ in $\mathcal{T}$, we define an automorphism $\delta_{Y}: Y \rightarrow Y$ such that $\delta_{X}=\phi(X)^{-1} \operatorname{Id}_{X}$ for $X \in \Lambda$ and $\delta_{Z}=\operatorname{Id}_{Z}$ for stalk complexes $Z$. Using $\delta$ as the adjusting isomorphisms, we obtain a new normalized pseudo-identity $\left(F^{\prime}, \omega^{\prime}\right)$ which is isomorphic to $(F, \omega)$ as triangle functors, and which satisfies $F^{\prime}\left(\right.$ inc $\left._{X}\right)=$ inc $_{X}$. We claim that $F^{\prime}\left(i_{m, n}\right)=i_{m, n}$. Indeed, this follows by applying $F^{\prime}$ to

$$
i_{m, n} \circ \operatorname{inc}_{X_{m, n}}=\operatorname{inc}_{X_{m-1, n}} .
$$

By the same argument, we infer that $F^{\prime}$ acts on $\left\{j_{m, n, a}, i_{m, n, b}^{\prime}, \iota_{m, n, a, b}, \xi_{m, a, b}\right\}$ by the identity. Therefore, $F^{\prime}$ acts on all inclusions by the identity.

Step 2 By Step 1, up to adjustment, we may assume that the normalized pseudoidentity $(F, \omega)$ acts trivially on all the inclusions. For $m<n$, we consider the connection $c_{m, n-1, n-1}: X_{m, n-1} \rightarrow X_{n-1, n-1}$. We observe that

$$
F\left(c_{m, n-1, n-1}\right)=c_{m, n-1, n-1} .
$$

We denote by $\phi: P_{0} \rightarrow P_{0}$ the unique morphism with $\phi\left(e_{0}\right)=\alpha_{N-1} \cdots \alpha_{1} \alpha_{0}$. We have that $\Sigma^{1-n}(\phi)$ equals the composition

$$
X_{n-1, n-1} \stackrel{\text { inc }}{\longrightarrow} X_{m, n-1} \stackrel{c_{m, n-1, n-1}}{\longrightarrow} X_{n-1, n-1} .
$$

By $F \Sigma^{1-n}(\phi)=\Sigma^{1-n}(\phi)$, we infer (4.1).

We assume that $F\left(\pi_{m, n}\right)=\lambda \pi_{m, n}$ for a nonzero scalar $\lambda$. We consider the following exact triangle in $\mathcal{T}$

$$
X_{n, n} \stackrel{\text { inc }}{\longrightarrow} X_{m, n} \stackrel{\pi_{m, n}}{\longrightarrow} X_{m, n-1} \stackrel{c_{m, n-1, n-1}}{\longrightarrow} X_{n-1, n-1} .
$$

Applying $(F, \omega)$ to this triangle and using the above observation, we have an exact triangle

$$
X_{n, n} \stackrel{\text { inc }}{\longrightarrow} X_{m, n} \stackrel{\lambda \pi_{m, n}}{\longrightarrow} X_{m, n-1} \stackrel{c_{m, n-1, n-1}}{\longrightarrow} X_{n-1, n-1} .
$$

Here, we use the fact that $\omega_{X_{n, n}}$ is the identity, since $(F, \omega)$ is normalized. By Lemma 2.4 we infer that $\lambda=1$ and thus $F\left(\pi_{m, n}\right)=\pi_{m, n}$ for any $m<n$.

Step 3 We claim that $F$ acts trivially on $\left\{\pi_{m, n, a}^{\prime}, p_{m, n, b}, q_{m, n, a, b}, \zeta_{m, a, b}\right\}$. Consequently, $F$ acts trivially on all the projections. 
To see $F\left(\pi_{m, n, a}^{\prime}\right)=\pi_{m, n, a}^{\prime}$, we apply $(F, \omega)$ to the following exact triangle

$$
X_{n, n} \stackrel{\text { inc }}{\longrightarrow} L_{m, n, a} \stackrel{\pi_{m, n, a}^{\prime}}{\longrightarrow} L_{m, n-1, a} \stackrel{c_{1}}{\longrightarrow} X_{n-1, n-1},
$$

where $c_{1}$ is the obvious connection. Similar to (4.1), we have $F\left(c_{1}\right)=c_{1}$. Applying Lemma 2.4 to the resulted exact triangle, we have the required identity.

To see $F\left(p_{m, n, b}\right)=p_{m, n, b}$, we apply $(F, \omega)$ to the following exact triangle

$$
L_{n, n, b} \stackrel{\text { inc }}{\longrightarrow} R_{m, n, b} \stackrel{p_{m, n, b}}{\longrightarrow} X_{m, n-1} \stackrel{c_{2}}{\longrightarrow} L_{n-1, n-1, b},
$$

where $c_{2}$ is the obvious connection. We have $F\left(c_{2}\right)=c_{2}$, which is similar to (4.1). Then the resulted exact triangle is as follows

$$
L_{n, n, b} \stackrel{\text { inc }}{\longrightarrow} R_{m, n, b} \stackrel{F\left(p_{m, n, b}\right)}{\longrightarrow} X_{m, n-1} \stackrel{c_{2}}{\longrightarrow} L_{n-1, n-1, b},
$$

Here, we use the fact that $\omega_{L_{n, n}, b}$ is the identity. Recall that $F\left(p_{m, n, b}\right)$ differs from $p_{m, n, b}$ by a nonzero scalar. Applying Lemma 2.4, we are done.

Similarly, we apply $(F, \omega)$ to

$$
L_{n, n, b} \stackrel{\text { inc }}{\longrightarrow} B_{m, n, a, b} \stackrel{q_{m, n, a, b}}{\longrightarrow} L_{m, n-1, a} \stackrel{c_{m, n-1, n-1, a, b}}{\longrightarrow} L_{n-1, n-1, b},
$$

and

$$
L_{m+1, m+1, b} \stackrel{\text { inc }}{\longrightarrow} Z_{m, a, b} \stackrel{\zeta_{m, a, b}}{\longrightarrow} L_{m, m, a} \stackrel{c_{m, m, m, a, b}}{\longrightarrow} L_{m, m, b},
$$

to deduce $F\left(q_{m, n, a, b}\right)=q_{m, n, a, b}$ and $F\left(\zeta_{m, a, b}\right)=\zeta_{m, a, b}$, respectively. This completes the proof of the claim.

We observe that $F\left(c_{l, m, n, a, b}\right)=c_{l, m, n, a, b}$. The case $m=n$ is similar to (4.1). Assume that $m<n$. We apply $F$ to the composition

$$
\Sigma^{-m}(\phi)=X_{m, m} \stackrel{\text { inc }}{\longrightarrow} L_{l, m, a} \stackrel{c_{l, m, n, a, b}}{\longrightarrow} R_{m, n, b} \stackrel{\mathrm{pr}}{\longrightarrow} X_{m, m} .
$$

Here, $\phi$ denotes the unnamed arrow $P_{0} \rightarrow P_{0}$. We have $F \Sigma^{-m}(\phi)=\Sigma^{-m}(\phi)$. Since $F$ acts trivially on all the inclusions and projections, we infer the required identity. Consequently, $F$ acts trivially on all the connections.

Step 4 We keep the assumptions in Step 2. We claim that $F(f)=f$ for any morphism $f$ of the mixed type. Consequently, $F$ acts trivially on the spanning set in Proposition 4.3 (1), as required.

We verify the claim for the 11 classes of morphisms. The idea is to compose those morphisms with suitable inclusions, and to use the fact that $F$ acts trivially on inclusions and connections.

Consider the morphism $L_{m, n, a} \rightarrow L_{m^{\prime}, n, b}$ of class (mx.I). We apply $F$ to the following commutative diagram

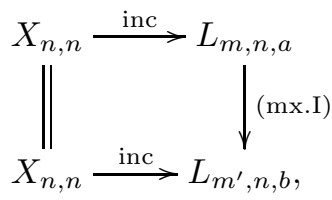

and deduce that $F$ acts trivially on the required morphism. The same argument works for (mx.II). For a morphism of class (mx.III), we apply $F$ to the following commutative diagram

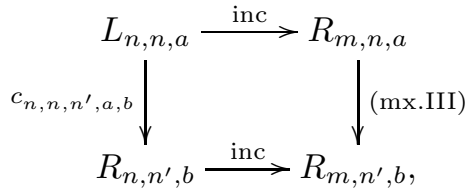


and use $F\left(c_{n, n, n^{\prime}, a, b}\right)=c_{n, n, n^{\prime}, a, b}$. Here, we have to observe that the composition in the diagram is nonzero. The same argument works for all the remaining 8 cases.

\section{The CASE $r>1$}

In this section, we prove Theorem 3.5 for the case $r>1$ and $N>r$. The idea of the proof is the same as in Section 4 , so we omit some details.

Let $A=A(r, N), \mathcal{A}=A$-proj and $\mathcal{T}=\mathbf{K}^{b}(\mathcal{A})$. We use Convention $(\dagger)$ in Section 3. In particular, the lower indices $s$ of $P_{s}$ are taken in $\mathbb{Z} / r \mathbb{Z}$. We consider the following objects in $\mathcal{T}$ :

$$
\begin{aligned}
X_{s, m, n} & =\cdots \rightarrow 0 \rightarrow P_{s} \rightarrow P_{s-1} \rightarrow \cdots \rightarrow P_{s-n+m+1} \rightarrow P_{s-n+m} \rightarrow 0 \rightarrow \cdots \\
L_{m, n, a} & =\cdots \rightarrow 0 \rightarrow Q_{a} \rightarrow P_{r-1} \rightarrow P_{r-2} \rightarrow \cdots \rightarrow P_{r-n+m+1} \rightarrow P_{r-n+m} \rightarrow 0 \rightarrow \cdots \\
R_{m, n, b} & =\cdots \rightarrow 0 \rightarrow P_{n-m-1} \rightarrow P_{n-m-2} \rightarrow \cdots \rightarrow P_{1} \rightarrow P_{0} \rightarrow Q_{b} \rightarrow 0 \rightarrow \cdots \\
B_{m, n, a, b} & =\cdots \rightarrow 0 \rightarrow Q_{a} \rightarrow P_{r-1} \rightarrow P_{r-2} \rightarrow \cdots \rightarrow P_{1} \rightarrow P_{0} \rightarrow Q_{b} \rightarrow 0 \rightarrow \cdots \\
Z_{m, a, b} & =\cdots \rightarrow 0 \rightarrow Q_{a} \rightarrow Q_{b} \rightarrow 0 \rightarrow \cdots .
\end{aligned}
$$

The supports of $X_{s, m, n}, L_{m, n, a}$ and $R_{m, n, b}$ are $[m, n]$ for $m \leq n$, where $s$ lies in $\mathbb{Z} / r \mathbb{Z}$, and $a, b$ run from $r$ to $N-1$. We observe that $X_{s, m, m}=\Sigma^{-m}\left(P_{s}\right)$ and $L_{m, m, a}=R_{m, m, a}=\Sigma^{-m}\left(Q_{a}\right)$. For $m<n-r$ with $r \mid(n-m-1)$ and $r \leq a, b<N$, the complex $B_{m, n, a, b}$ is defined, whose support is $[m, n]$. The complex $Z_{m, a, b}$ is supported in $[m, m+1]$, where $r \leq b<a<N$.

The following result is analogous to Lemma 4.1 .

Lemma 5.1. Keep the above notation. Then

$$
\Lambda=\left\{X_{s, m, n}, L_{m, n, a}, R_{m, n, b}, B_{m, n, a, b}, Z_{m, a, b}\right\}
$$

is a complete set of representatives of isoclasses of indecomposable objects in $\mathcal{T}$. Moreover, for each object $Y$ in $\Lambda$, we have $\operatorname{End}_{\mathcal{T}}(Y)=k \operatorname{Id}_{Y}$.

There are four types of morphisms between objects in $\Lambda$ : inclusions, projections, connections and morphisms of the mixed type; compare [1] and Section 4.

The first type is induced by the obvious inclusion: $i_{s, m, n}: X_{s, m, n} \rightarrow X_{s+1, m-1, n}$, $j_{m, n, a}: X_{r-1, m, n} \rightarrow L_{m-1, n, a}, i_{m, n, b}^{\prime}: R_{m, n, b} \rightarrow R_{m-1, n, b}$ for $s \in \mathbb{Z} / r \mathbb{Z}, m \leq n$ and $r \leq a, b<N ; \iota_{m, n, a, b}: R_{m, n, b} \rightarrow B_{m-1, n, a, b}$ for $m \leq n-r$ with $r \mid(n-m)$ and $r \leq a, b<N ; \xi_{m, a, b}: L_{m, m, b} \rightarrow Z_{m-1, a, b}$ for each integer $m$ and $r \leq b<a<N$. We will call any composition of these morphisms an inclusion, which will be denoted by "inc".

The second type is induced by the obvious projection: $\pi_{s, m, n}: X_{s, m, n} \rightarrow X_{s, m, n-1}$, $\pi_{m, n, a}^{\prime}: L_{m, n, a} \rightarrow L_{m, n-1, a}$ and $p_{m, n, b}: R_{m, n, b} \rightarrow X_{n-m-1, m, n-1}$ for $s \in \mathbb{Z} / r \mathbb{Z}$, $m<n$ and $r \leq a, b<N ; q_{m, n, a, b}: B_{m, n, a, b} \rightarrow L_{m, n-1, a}$ for $m<n-r$ with $r \mid(m-n-1)$ and $r \leq a, b<N ; \zeta_{m, a, b}: Z_{m, a, b} \rightarrow L_{m, m, a}$ for each integer $m$ and $r \leq b<a<N$. We will call any composition of these morphisms a projection, which will be denoted by "pr".

For the third type, we denote by $c_{l, m, n, a, b}: L_{l, m, a} \rightarrow R_{m, n, b}$ the following morphism

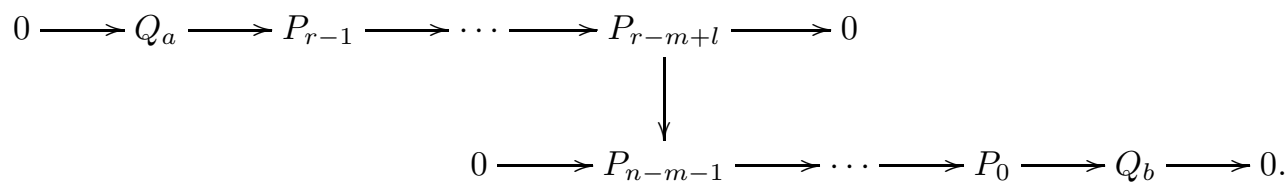

for $l \leq m \leq n$ satisfying $r \mid(n-l)$ and $r \leq a, b<N$. Here, the unique nonzero vertical map is the unnamed arrow $P_{r-m+l} \rightarrow P_{n-m-1}$, where we use the fact $r \mid(n-l)$. If $l=m<n$, the vertical map is given by $Q_{a} \rightarrow P_{r-1}$; if $l<m=n$, it is given by $P_{0} \rightarrow Q_{b}$. If $l=m=n$, we have to assume that $b<a$, in which case we 
have $c_{l, l, l, a, b}=\Sigma^{-l}(\phi)$, where $\phi: Q_{a} \rightarrow Q_{b}$ is the unnamed arrow. We will call any morphism of the form

$$
\operatorname{pr} \circ c_{l, m, n, a, b} \circ \text { inc }
$$

a connection.

The fourth type, called the mixed type, is divided into 11 classes of morphisms. They are listed as follows.

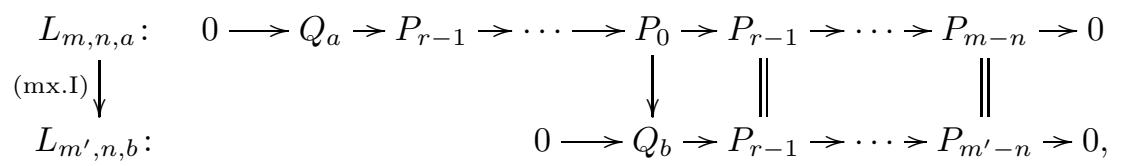

for $m<m^{\prime}<n$ with $r \mid\left(m^{\prime}-m\right)$ and $r \leq a, b<N$;

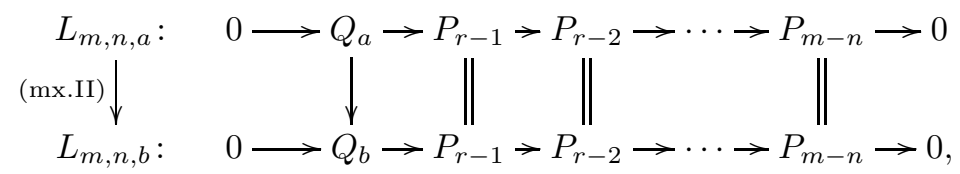

for $m<n$ and $r \leq b<a<N$;

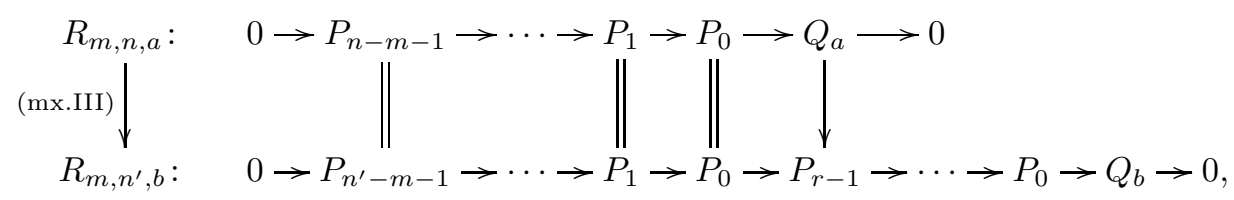

for $m<n<n^{\prime}$ with $r \mid\left(n^{\prime}-n\right)$ and $r \leq a, b<N$;

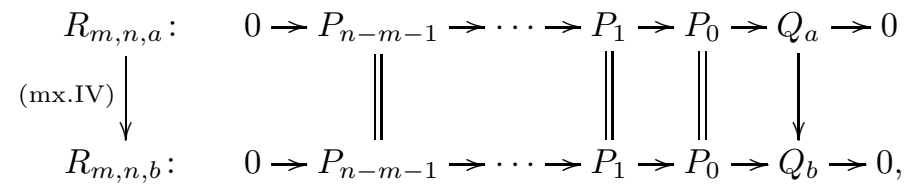

for $m<n$ and $r \leq b<a<N$;

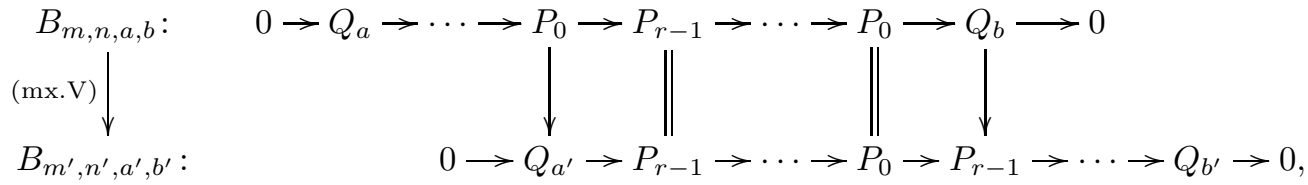

for $m<m^{\prime}<n<n^{\prime}$ with $r\left|\left(m^{\prime}-m\right), r\right|\left(n^{\prime}-n\right)$ and $r \leq a, b, a^{\prime}, b^{\prime}<N$;

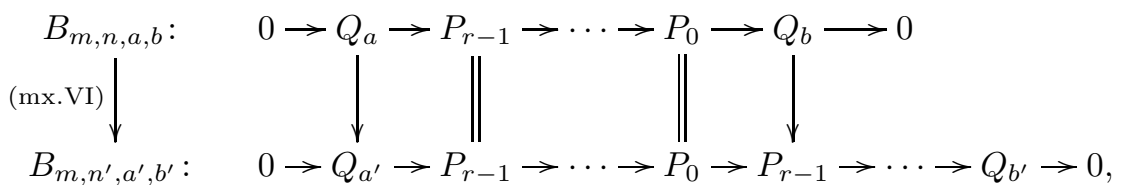

for $m+r<n<n^{\prime}$ with $r|(n-m-1), r|\left(n^{\prime}-n\right)$ and $r \leq a, b, a^{\prime}, b^{\prime}<N$ satisfying $a^{\prime} \leq a$, where for the case $a=a^{\prime}$ the unnamed arrow $Q_{a} \rightarrow Q_{a^{\prime}}$ is the identity map; dually, we have

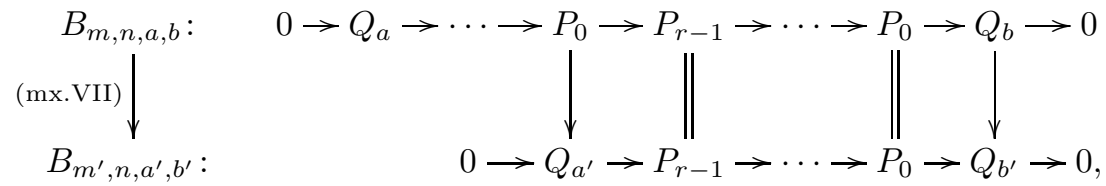


for $m<m^{\prime}<n-r$ with $r\left|\left(m^{\prime}-m\right), r\right|(n-m-1)$ and $r \leq a, b, a^{\prime}, b^{\prime}<N$ satisfying $b^{\prime} \leq b$, where for the case $b=b^{\prime}$ the unnamed arrow $Q_{b} \rightarrow Q_{b^{\prime}}$ is the identity map;

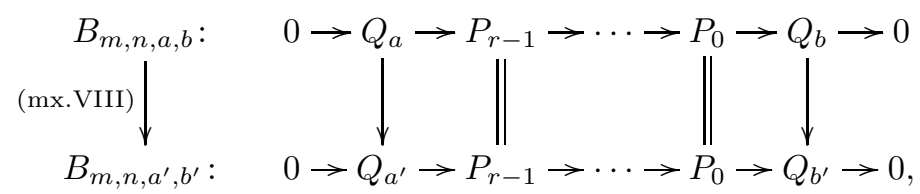

for $m<n-r$ with $r \mid(n-m-1), a^{\prime} \leq a$ and $b^{\prime} \leq b$;

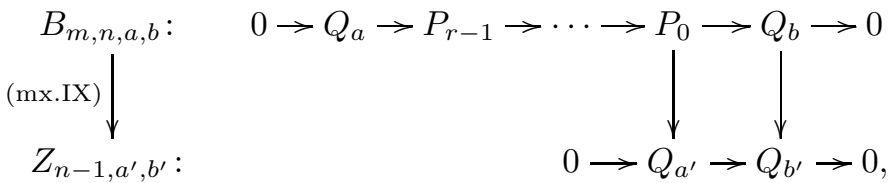

for $m<n-r$ with $r \mid(n-m-1)$ and $r \leq b^{\prime} \leq b<a^{\prime}<N$;

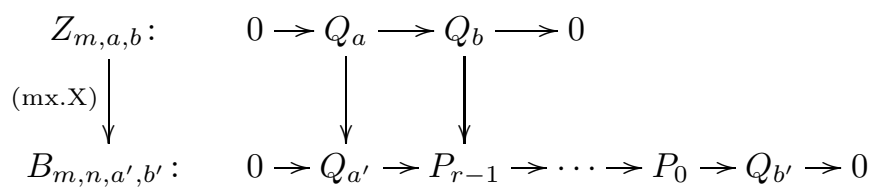

for $m \leq n-r$ with $r \mid(n-m-1)$ and $r \leq b<a^{\prime} \leq a<N$;

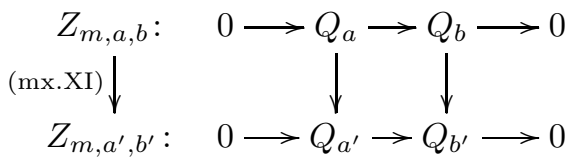

for each integer $m$ and $r \leq b^{\prime} \leq b<a^{\prime} \leq a<N$.

The following result can be proved by the same argument as in Proposition 4.3 . The second statement is contained in [5, Theorem 6.1].

Proposition 5.2. Keep the notation as above. Then the following statements hold.

(1) The above morphisms

$$
\begin{aligned}
& \left\{i_{s, m, n}, j_{m, n, a}, i_{m, n, b}^{\prime}, \iota_{m, n, a, b}, \xi_{m, a, b}\right\} \cup\left\{\pi_{s, m, n}, \pi_{m, n, a}^{\prime}, p_{m, n, b}, q_{m, n, a, b}, \zeta_{m, a, b}\right\} \\
& \cup\left\{c_{l, m, n, a, b}\right\} \cup\{(\mathrm{mx} . \mathrm{I})-(\mathrm{mx} . \mathrm{XI})\}
\end{aligned}
$$

form a spanning set of $\mathcal{T}$.

(2) For two objects $X, Y \in \Lambda$, we have $\operatorname{dim} \operatorname{Hom}_{\mathcal{T}}(X, Y) \leq 1$.

We now sketch a proof of Theorem 3.5 for the case $r>1$, which is almost identical to the one in Section 4. We only indicate necessary changes.

Proof. Let $(F, \omega)$ be a pseudo-identity on $\mathcal{T}$. By Lemma 3.2, we assume that $(F, \omega)$ is normalized.

It suffices to adjust $(F, \omega)$ by nonzero scalars such that the adjusted pseudoidentity $(\bar{F}, \bar{\omega})$ acts trivially on the spanning set in Proposition $5.2(1)$. We observe that Proposition 2.6 applies to $\mathcal{T}$, where the subset $S$ of $\Lambda=\operatorname{ind} \mathcal{T}$ is empty. Thanks to Proposition 2.6 (1), the homomorphism (3.1) is injective. By Lemma 2.2 and Proposition 2.6 (2), we infer that $(\bar{F}, \bar{\omega})$ is isomorphic to the identity functor. Therefore, the category $\mathcal{A}$ is strongly $\mathbf{K}$-standard.

For the required adjustment, we proceed as in Section 4 . The first step is almost the same, just replacing $X_{m, n}$ by $X_{s, m, n}$. Consequently, the adjusted pseudoidentity is still normalized and acts trivially on all the inclusions.

For the second step, (4.1) is replaced by $F\left(c_{s, m, n-1, n-1}\right)=c_{s, m, n-1, n-1}$. Here,

$$
c_{s, m, n-1, n-1}: X_{s, m, n-1} \longrightarrow X_{s-n+m, n-1, n-1}
$$


is the obvious connection, whose $(n-1)$-th degree is given by the unnamed arrow $P_{s-n+m+1} \rightarrow P_{s-n+m}$. Then the triangle (4.2) is replaced by the following one

$$
X_{s-n+m, n, n} \stackrel{\text { inc }}{\longrightarrow} X_{s, m, n} \stackrel{\pi_{s, m, n}}{\longrightarrow} X_{s, m, n-1} \stackrel{c_{s, m, n-1, n-1}}{\longrightarrow} X_{s-n+m, n-1, n-1} .
$$

Applying $(F, \omega)$ to it and using Lemma 2.4, we infer that $F\left(\pi_{s, m, n}\right)=\pi_{s, m, n}$. Here, we use the fact that $\omega_{X_{s-n+m, n, n}}$ is the identity, since the given pseudo-identity is normalized. The remaining part of Step 2 carries over as in Section 4 . The third step is the same as Step 3 in Section 4.

For the last step, we just repeat Step 4 in Section 4. For example, the commutative diagram (4.3) is replaced by

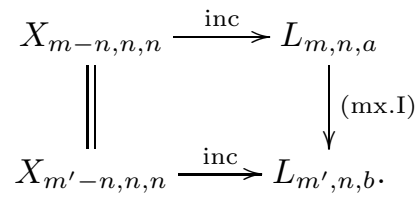

Here, we recall that $r \mid\left(m^{\prime}-m\right)$ in defining (mx.I), and thus $X_{m-n, n, n}=X_{m^{\prime}-n, n, n}$. We omit the details.

Acknowledgements The authors are supported by National Natural Science Foundation of China (No.s 11522113, 11671245 and 11601098), and Natural Science Foundation of Guizhou Province (QSF[2016]1038).

\section{REFERENCES}

[1] K.K. Arnesen, R. Laking, and D. Pauksztello, Morphisms between indecomposable complexes in the bounded derived category of a gentle algebra, J. Algebra 467 (2016), $1-46$.

[2] V. Bekkert, and H. Merklen, Indecomposables in derived categories of gentle algebras, Algebr. Represent. Theory 6 (2003), 285-302.

[3] G. Bobinski, The graded centers of derived discrete algebras, J. Algebra 333 (2011), 55-66.

[4] G. Bobinski, C. Geiss, And A. Skowronski, Classification od discrete derived categories, Cent. Eur. J. Math. 2 (2004), 19-49.

[5] N. Broomhead, D. Pauksztello, and D. Ploog, Discrete derived categories I, Homomorphisms, autoequivalences and t-structures, Math. Z. 285 (1) (2017), 39-89.

[6] X.W. Chen, A note on standard equivalences, Bull. London Math. Soc. 48 (2016), 797-801.

[7] X.W. Chen, And C.M. Ringel, Hereditary triangulated categories, J. Noncomm. Geometry, to appear,

[8] X.W. Chen, And Y. Ye, The D-standard and K-standard categories, arXiv: 1612.06051.

[9] D. Happel, Triangulated Categories in the Representation Theory of Finite Dimensional Algebras, London Math. Soc. Lecture Notes Ser. 119, Cambridge Univ. Press, Cambridge, 1988.

[10] S. Koenig, And D. YAng, Silting objects, simple-minded collections, t-structures and cot-structures for finite-dimensional algebras, Doc. Math. 19 (2014), 403-438.

[11] M. Kunzer, On the center of the derived caregory, preprint, 2006.

[12] H. Minamoto, Ampleness of two-sided tilting complexes, Inter. Math. Res. Not. IMRN 1 (2012), 67-101.

[13] J.I. Miyachi, And A. Yekutieli, Derived Picard groups of finite-dimensional hereditary algebras, Compo. Math. 129 (2001), 341-368.

[14] J. Rickard, Derived equivalences as derived functors, J. London Math. Soc. (2) 43 (1991), $37-48$.

[15] D. Vossieck, The algebras with discrete derived category, J. Algebra 243 (1) (2001), 168176.

[16] A. Zimmermann, Representation Theory, A Homological Algebra Point of View, Springer, International Publishing Switzerland, 2014.

Xiao-Wu Chen

Key Laboratory of Wu Wen-Tsun Mathematics, Chinese Academy of Sciences 
School of Mathematical Sciences, University of Science and Technology of China

No. 96 Jinzhai Road, Hefei, 230026, Anhui, P.R. China.

URL: http://home.ustc.edu.cn/ ${ }^{\sim}$ xwchen

Chao Zhang

Department of Mathematics, Guizhou University, Guiyang 550025, Guizhou, P.R. China. 